\title{
ON GENERALIZED TWO-INDEX HERMITE MATRIX POLYNOMIALS
}

\author{
LEVENT KARGIN AND VELI KURT
}

Received 30 September, 2015

\begin{abstract}
In this study, we give a new generalization for Hermite matrix polynomials. We obtain some formulas related to an explicit representation, matrix recurrence relations and the Rodrigues formula for two-index Hermite matrix polynomials. Also we generalize Runge's addition formula and Nielsen's identity for these new generalizations. Moreover we give Burchnall's operational formula and Nielsen's identity for Hermite matrix polynomials by means of two-index Hermite matrix polynomials.
\end{abstract}

2010 Mathematics Subject Classification: 15A60; 33C45; 33E20

Keywords: Hermite matrix polynomials, operators

\section{INTRODUCTION AND NOTATION}

Throughout this paper, if $A$ is a matrix in $\mathbb{C}^{r \times r}$, its spectrum $\sigma(A)$ denotes the set of all eigenvalues of $A$. Its 2 -norm is denoted by $\|A\|$ and defined by ([10])

$$
\|A\|=\sup _{x \neq 0} \frac{\|A x\|_{2}}{\|x\|_{2}}
$$

where for a vector $y$ in $\mathbb{C}^{r},\|y\|_{2}=\left(y^{T}, y\right)^{\frac{1}{2}}$ is the Euclidean norm of $y$. Moreover the zero matrix and identity matrix will be denoted by 0 and $I$, respectively. For a matrix $A(k, n)$ in $\mathbb{C}^{r \times r}$ for $n \geq 0$ and $k \geq 0$, then in an analogous way to the proof of Lemma 11 of [26], it follows that

$$
\sum_{n=0}^{\infty} \sum_{k=0}^{\infty} A(k, n)=\sum_{n=0}^{\infty} \sum_{k=0}^{n} A(k, n-k) .
$$

If $A$ is a matrix in $\mathbb{C}^{r \times r}$ such that $\operatorname{Re}(z)>0$ for every eigenvalue $z \in \sigma(A)$, we say a positive stable matrix. Also throughout this paper, we will use a positive stable matrix $A$ in $\mathbb{C}^{r \times r}$. Then Hermite matrix polynomials are defined by means of the 
following generating function [13]

$$
\sum_{n=0}^{\infty} H_{n}(x, A) \frac{t^{n}}{n !}=\exp \left(x t \sqrt{2 A}-t^{2} I\right) .
$$

From the above equation we have

$$
H_{n}(x, A)=\sum_{k=0}^{\left[\frac{n}{2}\right]} \frac{(-1)^{k} n !(x \sqrt{2 A})^{n-2 k}}{k !(n-2 k) !}, \quad n \geq 0
$$

and

$$
\frac{d^{k}}{d x^{k}} H_{n}(x, A)=(\sqrt{2 A})^{k} \frac{n !}{(n-k) !} H_{n-k}(x, A), \quad 0 \leq k \leq n .
$$

Also, Hermite matrix polynomials satisfy Runge's addition formula ([1])

$$
2^{\frac{n}{2}} H_{n}(x+y, A)=\sum_{k=0}^{n}\left(\begin{array}{l}
n \\
k
\end{array}\right) H_{k}(\sqrt{2} x, A) H_{n-k}(\sqrt{2} y, A),
$$

a generating matrix function ([14])

$$
\sum_{n=0}^{\infty} H_{n}(x, A) H_{n}(y, A) \frac{t^{n}}{n !}=\left(1-4 t^{2}\right)^{-\frac{1}{2}} \exp \left(\frac{2 A\left(x y t-\left(x^{2}+y^{2}\right) t^{2}\right)}{1-4 t^{2}}\right)
$$

and the summation formula ([17])

$$
H_{n}(x, A) H_{m}(x, A)=m ! n ! \sum_{k=0}^{\min (m, n)} \frac{2^{k} H_{m+n-2 k}(x, A)}{(m-k) !(n-k) ! k !} .
$$

Some other properties of Hermite matrix polynomials can be found in $[1,3,7,15$, 22, 29,33].

In the recent two decades, special matrix functions are an emergent field whose development is reaching important results both the theoretical and practical points of view. Some important results for classical scalar special functions are generalized to special matrix functions, see for example [2, 5, 8, 9, 16, 21, 30, 31]. Also, some generalized form of these matrix polynomials are introduced and studied in $[4,18-20,23-25,27,28,32]$. Our purpose here is to introduce and study a new generalization which is called two-index Hermite matrix polynomials. The organization of the paper is as follows. In Section 2, the class of two-index Hermite matrix polynomials is introduced. Starting from an appropriate matrix generating function, matrix recurrence relations and Runge's additional theorem and multiplication theorem for these matrix polynomials are established. Section 3 deals with the operational identity which simplifies the studying properties of two-index Hermite matrix polynomials rather than generating matrix function. In the sense of that we construct Rodrigues formula. We give Nielsen's identity for these matrix polynomials as an 
application of this formula. Finally, we focus on Hermite matrix polynomials. We establish Burchnall's operational formula and Nielsen's identity.

\section{Two-Index Hermite Matrix Polynomials: Definition AND PROPERTIES}

One of the most direct ways of exploring generalized classes of Hermite matrix polynomials is to start from modifying the form of the ordinary generating function. So, we define two-index Hermite polynomials $H_{n, m}(x, A)$ by

$$
\begin{aligned}
F(x, u, v, A) & =\exp \left(\sqrt{\frac{A}{2}} x(u+v)-u v I\right) \\
& =\sum_{n=0}^{\infty} \sum_{m=0}^{\infty} H_{n, m}(x, A) \frac{u^{n}}{n !} \frac{v^{m}}{m !}, \quad|u|<\infty,|v|<\infty .
\end{aligned}
$$

We can write

$$
\exp \left(\sqrt{\frac{A}{2}} x(u+v)-u v I\right)=\sum_{n=0}^{\infty} \sum_{m=0}^{\infty} \sum_{k=0}^{\infty}(-1)^{k}\left(x \sqrt{\frac{A}{2}}\right)^{n+m} \frac{u^{n+k} v^{m+k}}{k ! n ! m !} .
$$

Using the relation (1.1) yields

$$
\begin{aligned}
& \exp \left(\sqrt{\frac{A}{2}} x(u+v)-u v I\right) \\
&=\sum_{n=0}^{\infty} \sum_{m=0}^{\infty} \sum_{k=0}^{\min (m, n)}(-1)^{k}\left(\begin{array}{l}
m \\
k
\end{array}\right)\left(\begin{array}{l}
n \\
k
\end{array}\right) k !\left(x \sqrt{\frac{A}{2}}\right)^{m+n-2 k} \frac{u^{n} v^{m}}{n ! m !} .
\end{aligned}
$$

From (2.1) and (2.2), we obtain an explicit representation for the two-index Hermite matrix polynomials in the form:

$$
H_{n, m}(x, A)=\sum_{k=0}^{\min (m, n)}(-1)^{k}\left(\begin{array}{c}
m \\
k
\end{array}\right)\left(\begin{array}{l}
n \\
k
\end{array}\right) k !\left(x \sqrt{\frac{A}{2}}\right)^{m+n-2 k} .
$$

It follows that $H_{n, m}(x, A)$ is a polynomial of degree $m+n$ and $H_{n, m}(x, A)=$ $H_{m, n}(x, A)$. For the unity of the formulations, we define trivially

$$
H_{n, m}(x, A)=\mathbf{0},
$$

whenever $m<0$ or $n<0$. Note that $H_{n, 0}(x, A)=\left(x \sqrt{\frac{A}{2}}\right)^{n}$. From (2.3), it is clear that

$$
H_{n, m}(0, A)=\left\{\begin{array}{cc}
0 & ; m \neq n \\
(-1)^{n} n ! I & ; m=n
\end{array}\right.
$$


and

$$
H_{1, m}(x, A)=\left(x \sqrt{\frac{A}{2}}\right)^{m+1}-m\left(x \sqrt{\frac{A}{2}}\right)^{m-1},
$$

for every integer $m \geq 1$.

The first few values of $H_{n, m}(x, A)$ are given by

\begin{tabular}{|c|c|c|}
\hline$H_{n, m}(x, A)$ & $m=1$ & $m=2$ \\
\hline$n=1$ & $\left(x \sqrt{\frac{A}{2}}\right)^{2}-I$ & $\left(x \sqrt{\frac{A}{2}}\right)^{3}-2\left(x \sqrt{\frac{A}{2}}\right)$ \\
\hline$n=2$ & $\left(x \sqrt{\frac{A}{2}}\right)^{3}-2\left(x \sqrt{\frac{A}{2}}\right)$ & $\left(x \sqrt{\frac{A}{2}}\right)^{4}-4\left(x \sqrt{\frac{A}{2}}\right)^{2}+2 I$ \\
\hline$n=3$ & $\left(x \sqrt{\frac{A}{2}}\right)^{4}-3\left(x \sqrt{\frac{A}{2}}\right)$ & $\left(x \sqrt{\frac{A}{2}}\right)^{5}-6\left(x \sqrt{\frac{A}{2}}\right)^{3}+6\left(x \sqrt{\frac{A}{2}}\right)$ \\
\hline
\end{tabular}

From (2.3), one can deduce easily the symmetry formula

$$
H_{n, m}(-x, A)=(-1)^{n+m} H_{n, m}(x, A) .
$$

So that the $H_{n, m}(x, A)$ is odd or even if and only if $n+m$ is odd or even respectively. By taking $u=v$ in (2.1), we obtain a relation between Hermite matrix polynomials and our generalization as

$$
H_{n}(x, A)=\sum_{m=0}^{n}\left(\begin{array}{l}
n \\
m
\end{array}\right) H_{n-m, m}(x, A) .
$$

Now we study the uniform convergence of the matrix series (2.1) in the complex domain $|x|<a$ for a fixed value of $u$ and $v$. By taking 2-norm in (2.3), we get

$$
\left\|H_{n, m}(x, A)\right\| \leq H_{n, m}\left(a\left\|\sqrt{\frac{A}{2}}\right\|\right),
$$

where $H_{n, m}(x)$ is the classical scalar two-index Hermite polynomials in the form ([12])

$$
H_{n, m}(x)=\sum_{q=0}^{\min (m, n)}(-1)^{q} q !\left(\begin{array}{c}
m \\
q
\end{array}\right)\left(\begin{array}{l}
n \\
q
\end{array}\right) x^{m+n-2 q} .
$$

Moreover

$$
\exp \left(\left\|\sqrt{\frac{A}{2}}\right\| a|u+v|+|u||v|\right)=\sum_{n=0}^{\infty} \sum_{m=0}^{\infty} H_{n, m}\left(a\left\|\sqrt{\frac{A}{2}}\right\|\right) \frac{|u|^{n}}{n !} \frac{|v|^{m}}{m !} .
$$

In view of (2.7) and (2.8), the matrix series (2.1) is termwise differentiable with respect to $x$. 
Proposition 1. The following recurrence formula for $H_{n, m}(x, A)$ holds

$$
\frac{d^{s}}{d x^{s}} H_{n, m}(x, A)=s !\left(\sqrt{\frac{A}{2}}\right)^{s} \sum_{r=0}^{s}\left(\begin{array}{c}
n \\
s-r
\end{array}\right)\left(\begin{array}{c}
m \\
r
\end{array}\right) H_{n-s+r, m-r}(x, A) .
$$

Proof. Differentiating the both sides of (2.1) with respect to $x$ and making appropriate changes of indices, we get

$$
\frac{d}{d x} H_{n, m}(x, A)=\sqrt{\frac{A}{2}}\left[n H_{n-1, m}(x, A)+m H_{n, m-1}(x, A)\right] .
$$

To end the proof we apply mathematical induction to (2.10).

Proposition 2. Two-index Hermite matrix polynomials satisfy the following recurrences formulas

$$
\begin{gathered}
H_{n+1, m}(x, A)=x \sqrt{\frac{A}{2}} H_{n, m}(x, A)-m H_{n, m-1}(x, A), \\
H_{n, m+1}(x, A)=x \sqrt{\frac{A}{2}} H_{n, m}(x, A)-n H_{n-1, m}(x, A), \\
(n-m) H_{n, m}(x, A)=x \sqrt{\frac{A}{2}}\left(H_{n+1, m}(x, A)-H_{n, m+1}(x, A)\right) .
\end{gathered}
$$

Proof. Taking termwise differentiation with respect to $u$ to (2.1), we have

$$
\frac{\partial}{\partial u} F(x, u, v, A)=\left(x \sqrt{\frac{A}{2}}-v I\right) F(x, u, v, A)
$$

and, so that

$$
\begin{aligned}
& \sum_{n=1}^{\infty} \sum_{m=0}^{\infty} n H_{n, m}(x, A) \frac{u^{n-1}}{n !} \frac{v^{m}}{m !} \\
& \quad=\sum_{n=0}^{\infty} \sum_{m=0}^{\infty} x \sqrt{\frac{A}{2}} H_{n, m}(x, A) \frac{u^{n}}{n !} \frac{v^{m}}{m !}-\sum_{n=0}^{\infty} \sum_{m=0}^{\infty} H_{n, m}(x, A) \frac{u^{n}}{n !} \frac{v^{m+1}}{m !} .
\end{aligned}
$$

Making appropriate changes of indices yields (2.11). For the proof of (2.12), we apply the same method after differentiating (2.1) with respect to $v$. Finally, subtracting (2.11) from (2.12) gives (2.13).

Proposition 3. Two-index Hermite matrix polynomials satisfy the multiplication and addition formulas as follow

$$
H_{n, m}(\mu x, A)=\mu^{m+n} \sum_{k=0}^{\min (n, m)}\left(\begin{array}{l}
n \\
k
\end{array}\right)\left(\begin{array}{l}
m \\
k
\end{array}\right) k !\left(1-\frac{1}{\mu^{2}}\right)^{k} H_{n-k, m-k}(x, A),
$$




$$
\begin{aligned}
\left(\lambda^{2}+\mu^{2}\right)^{\frac{n+m}{2}} & H_{n, m}\left(\frac{\lambda z_{1}+\mu z_{2}}{\left(\lambda^{2}+\mu^{2}\right)^{\frac{1}{2}}}, A\right) \\
& =\lambda^{m+n} \sum_{k=0}^{n} \sum_{l=0}^{m}\left(\begin{array}{l}
n \\
k
\end{array}\right)\left(\begin{array}{c}
m \\
l
\end{array}\right)\left(\frac{\mu}{\lambda}\right)^{k+l} H_{n-k, m-l}\left(z_{1}, A\right) H_{k, l}\left(z_{2}, A\right)
\end{aligned}
$$

where $\lambda$ and $\mu$ are any complex numbers.

Proof. Taking $\mu x$ for $x, \frac{u}{\mu}$ for $u$ and $\frac{v}{\mu}$ for $v$ in (2.1), we have

$$
\begin{aligned}
\sum_{n=0}^{\infty} \sum_{m=0}^{\infty} \frac{H_{n, m}(\mu x, A)}{\mu^{m+n}} \frac{u^{n}}{n !} \frac{v^{m}}{m !} & =\exp \left(\sqrt{\frac{A}{2}} x(u+v)-\frac{u v}{\mu^{2}} I\right) \\
& =\exp \left(\sqrt{\frac{A}{2}} x(u+v)-\left(u v+u v-\frac{u v}{\mu^{2}}\right) I\right)
\end{aligned}
$$

Then using (1.1) and comparing the coefficients of $\frac{u^{n}}{n !} \frac{v^{m}}{m !}$ in both sides of the identity, we get (2.15). Eq. (2.14) can be proved similarly.

Corollary 1. Two-index Hermite matrix polynomials satisfy the following equations:

$$
\begin{aligned}
2^{\frac{n+m}{2}} H_{n, m}(\sqrt{2} x, A) & =\sum_{k=0}^{n} \sum_{l=0}^{m}\left(\begin{array}{c}
n \\
k
\end{array}\right)\left(\begin{array}{c}
m \\
l
\end{array}\right) H_{n-k, m-l}(x, A) H_{k, l}(x, A) \\
2^{\frac{n+m}{2}} H_{n, m}(x+y, A) & =\sum_{k=0}^{n} \sum_{l=0}^{m}\left(\begin{array}{c}
n \\
k
\end{array}\right)\left(\begin{array}{c}
m \\
l
\end{array}\right) H_{n-k, m-l}(\sqrt{2} x, A) H_{k, l}(\sqrt{2} y, A) \\
2^{\frac{n+m}{2}} H_{n, m}(x, A) & =\sum_{k=0}^{n} \sum_{l=k}^{m}(-1)^{k} k !\left(\begin{array}{c}
n \\
k
\end{array}\right)\left(\begin{array}{c}
m \\
l
\end{array}\right) H_{n-k, m-l}(\sqrt{2} x, A)
\end{aligned}
$$

Equation (2.17) is the Runge's addition formula for two-index Hermite matrix polynomials.

\section{Some Results for Two-Index Hermite Matrix Polynomials}

In this section, we obtain an operational formula and give its applications such as Rodrigues formula and Nielsen's identity for two-index Hermite matrix polynomials. Moreover we establish Burchnall's operational formula and Nielsen's identity for Hermite matrix polynomials. Firstly, we have the following main theorem. 
Theorem 1. For $n, m \in \mathbb{N} \cup\{0\}$, we have the following operational formula

$$
H_{n, m}(x, A)=\left(x \sqrt{\frac{A}{2}}-\left(\sqrt{\frac{A}{2}}\right)^{-1} \frac{d}{d x}\right)^{n}\left(x \sqrt{\frac{A}{2}}\right)^{m} .
$$

Proof. It is clear that

$$
\begin{aligned}
\exp \left(-u\left(\sqrt{\frac{A}{2}}\right)^{-1} \frac{d}{d x}\right) e^{x \sqrt{\frac{A}{2}} v} & =\sum_{n=0}^{\infty} \frac{(-u)^{n}}{n !}\left(\sqrt{\frac{A}{2}}\right)^{-n} \frac{d^{n}}{d x^{n}} e^{x \sqrt{\frac{A}{2}} v} \\
& =e^{x \sqrt{\frac{A}{2}} v-u v I} .
\end{aligned}
$$

Multiplying both side of the above equation with $e^{x \sqrt{\frac{A}{2}} u}$, we get

$$
\exp \left[\left(x \sqrt{\frac{A}{2}}-\left(\sqrt{\frac{A}{2}}\right)^{-1} \frac{d}{d x}\right) u\right] e^{x \sqrt{\frac{A}{2}} v}=\exp \left(\sqrt{\frac{A}{2}} x(u+v)-u v I\right) .
$$

So we have

$$
\begin{gathered}
\sum_{n=0}^{\infty} \sum_{m=0}^{\infty}\left(x \sqrt{\frac{A}{2}}-\left(\sqrt{\frac{A}{2}}\right)^{-1} \frac{d}{d x}\right)^{n}\left(x \sqrt{\frac{A}{2}}\right)^{m} \frac{u^{n}}{n !} \frac{v^{m}}{m !} \\
=\sum_{n=0}^{\infty} \sum_{m=0}^{\infty} H_{n, m}(x, A) \frac{u^{n}}{n !} \frac{v^{m}}{m !} .
\end{gathered}
$$

Comparing the coefficients of $\frac{u^{n}}{n !} \frac{v^{m}}{m !}$ in both sides of the above equation, we get (3.1).

In the following corollary, we obtain another recurrence formula for the two-index Hermite matrix polynomials.

Corollary 2. For $n, m, r \in \mathbb{N} \cup\{0\}$, we have

$$
H_{n+r, m}(x, A)=\left(x \sqrt{\frac{A}{2}}-\left(\sqrt{\frac{A}{2}}\right)^{-1} \frac{d}{d x}\right)^{n} H_{r, m}(x, A) .
$$

For the proof of the Rodrigues formula and the Nielsen's identity for $H_{n, m}(x, A)$ we need the following lemma.

Lemma 1. For any $n$-times differentiable functions $f(x)$ and $g(x)$ we have

$$
\left(x \sqrt{\frac{A}{2}}-\left(\sqrt{\frac{A}{2}}\right)^{-1} \frac{d}{d x}\right)^{n}[f(x) g(x)]
$$




$$
=e^{\frac{A}{4} x^{2}}(-1)^{n}\left(\sqrt{\frac{A}{2}}\right)^{-n} \frac{d^{n}}{d x^{n}}\left[e^{-\frac{A}{4} x^{2}} f(x) g(x)\right] .
$$

Proof. One can prove (3.3) by mathematical induction.

Setting $f(x)=\left(x \sqrt{\frac{A}{2}}\right)^{m}$ and $g(x)=1$ in (3.3) we have the following corollary.

Corollary 3. The Rodrigues formula for $H_{n, m}(x, A)$ is given as follows:

$$
H_{n, m}(x, A)=e^{\frac{A}{4} x^{2}}(-1)^{n}\left(\sqrt{\frac{A}{2}}\right)^{m-n} \frac{d^{n}}{d x^{n}}\left[e^{-\frac{A}{4} x^{2}} x^{m}\right] .
$$

As a conclusion of the above corollary, it is good to note that two-index Hermite matrix polynomials constitute a subclass of matrix analogues of Gould-Hopper polynomials defined by ([11])

$$
H_{n}^{\gamma}(x, \alpha, p)=(-1)^{n} x^{-\alpha} e^{p x^{\gamma}} \frac{d^{n}}{d x^{n}}\left(x^{\alpha} e^{-p x^{\nu}}\right) .
$$

In fact, we have

$$
H_{n, m}(x, A)=x^{m}\left(\sqrt{\frac{A}{2}}\right)^{m-n} H_{n}^{2}\left(x, m, \frac{A}{4}\right) .
$$

Now, we give a formula that calculates the weighted sum of the product of the same polynomials in the following proposition.

Proposition 4. For $n, m, r \in \mathbb{N} \cup\{0\}$, the Nielsen's identity for $H_{n, m}(x, A)$ holds:

$$
\begin{aligned}
H_{n+r, m}(x, A)=(-1)^{n} \sum_{k, s, l=0}^{n, k, s}\left(\sqrt{\frac{A}{2}}\right)^{s-m-k} s !\left(\begin{array}{l}
n \\
k
\end{array}\right)\left(\begin{array}{c}
s \\
k
\end{array}\right)\left(\begin{array}{c}
r \\
s-l
\end{array}\right)\left(\begin{array}{c}
m \\
l
\end{array}\right)(m)_{n-k} \\
\times \frac{x^{k}}{x^{m+n}} H_{n-k, m}(x, A) H_{r-s+k, m-l}(x, A),
\end{aligned}
$$

where $(z)_{n}$ is Pochhammer symbol.

Proof. Taking $g(x)=\left(x \sqrt{\frac{A}{2}}\right)^{m}$ in (3.3), applying the Leibniz formula and using (3.4), we get

$$
\begin{aligned}
\left(x \sqrt{\frac{A}{2}}-\left(\sqrt{\frac{A}{2}}\right)^{-1} \frac{d}{d x}\right)^{n}\left[f(x)\left(x \sqrt{\frac{A}{2}}\right)^{m}\right] \\
=\sum_{k=0}^{n}(-1)^{k}\left(\begin{array}{l}
n \\
k
\end{array}\right)\left(\sqrt{\frac{A}{2}}\right)^{-k} H_{n-k, m}(x, A) \frac{d^{k}}{d x^{k}}[f(x)] .
\end{aligned}
$$


Then setting $f(x)=\left(x \sqrt{\frac{A}{2}}\right)^{-m} H_{r, m}(x, A)$ in (3.5) and using (3.2) we get

$$
\begin{aligned}
& H_{n+r, m}(x, A) \\
& \quad=\sum_{k=0}^{n}(-1)^{k}\left(\begin{array}{l}
n \\
k
\end{array}\right)\left(\sqrt{\frac{A}{2}}\right)^{-k} H_{n-k, m}(x, A) \frac{d^{k}}{d x^{k}}\left[\left(x \sqrt{\frac{A}{2}}\right)^{-m} H_{r, m}(x, A)\right] .
\end{aligned}
$$

Applying the Leibniz formula and using (2.9), we get the proof.

Now, we focus on Burchnall's operational formula and Nielsen's identity for Hermite matrix polynomials. Setting (3.1) in (2.6) we have

$$
\begin{aligned}
H_{n}(x, A) & =\sum_{m=0}^{n}\left(\begin{array}{c}
n \\
m
\end{array}\right) H_{n-m, m}(x, A) \\
& =\sum_{m=0}^{n}\left(\begin{array}{l}
n \\
m
\end{array}\right)\left(x \sqrt{\frac{A}{2}}-\left(\sqrt{\frac{A}{2}}\right)^{-1} \frac{d}{d x}\right)^{n-m}\left(x \sqrt{\frac{A}{2}}\right)^{m} .
\end{aligned}
$$

Thus we have the following corollary.

Corollary 4. Burchnall's operational formula for $H_{n}(x, A)$ holds

$$
H_{n}(x, A)=\left(x \sqrt{2 A}-\left(\sqrt{\frac{A}{2}}\right)^{-1} \frac{d}{d x}\right)^{n}(I) .
$$

In the following corollary, we obtain another recurrence formula for Hermite matrix polynomials.

Corollary 5. For $n, r \in \mathbb{N} \cup\{0\}$,

$$
H_{n+r}(x, A)=\left(x \sqrt{2 A}-\left(\sqrt{\frac{A}{2}}\right)^{-1} \frac{d}{d x}\right)^{n} H_{r}(x, A) .
$$

As an application of the above corollary, we state the Nielsen's identity for Hermite matrix polynomials in the following theorem.

Theorem 2. For $n, r \in \mathbb{N} \cup\{0\}$, we have the following equation

$$
H_{n+r}(x, A)=\sum_{k=0}^{\min (n, r)}(-2)^{k}\left(\begin{array}{l}
n \\
k
\end{array}\right)\left(\begin{array}{l}
r \\
k
\end{array}\right) k ! H_{n-k}(x, A) H_{r-k}(x, A) .
$$

Proof. Using mathematical induction one can show that

$$
\left(x \sqrt{2 A}-\left(\sqrt{\frac{A}{2}}\right)^{-1} \frac{d}{d x}\right)^{n}[f(x)]
$$




$$
=\sum_{k=0}^{n}(-1)^{k}\left(\begin{array}{l}
n \\
k
\end{array}\right)\left(\sqrt{\frac{A}{2}}\right)^{-k} H_{n-k}(x, A) \frac{d^{k}}{d x^{k}}[f(x)] .
$$

For $f(x)=H_{r}(x, A)$ in (3.9), we obtain

$$
H_{n+r}(x, A)=\sum_{k=0}^{n}(-1)^{k}\left(\begin{array}{l}
n \\
k
\end{array}\right)\left(\sqrt{\frac{A}{2}}\right)^{-k} H_{n-k}(x, A) \frac{d^{k}}{d x^{k}}\left[H_{r}(x, A)\right] .
$$

Therefore, using (1.3) and $H_{-1}(x, A)=\mathbf{0}$ we get

$$
H_{n+r}(x, A)=\sum_{k=0}^{\min (n, r)}(-2)^{k}\left(\begin{array}{l}
n \\
k
\end{array}\right)\left(\begin{array}{l}
r \\
k
\end{array}\right) k ! H_{n-k}(x, A) H_{r-k}(x, A) .
$$

From the above theorem we can immediately derive the following identity as a particular case

$$
H_{2 n}(x, A)=(-2)^{n}(n !)^{2} \sum_{k=0}^{n} \frac{(-1)^{k}\left[H_{k}(x, A)\right]^{2}}{2^{k}(k !)^{2}(n-k) !} .
$$

Moreover, the use of the binomial transform see for details ([6]) to the identity (3.10) gives the inverse of the relation (3.10). So, $\left[H_{n}(x, A)\right]^{2}$ can be written as

$$
\left[H_{n}(x, A)\right]^{2}=H_{n}(x, A) H_{n}(x, A)=(2)^{n}(n !)^{2} \sum_{k=0}^{n} \frac{H_{2 k}(x, A)}{2^{k}(k !)^{2}(n-k) !} .
$$

It is good to note that the above equation is the special case of (1.4).

\section{ACKNOWLEDGEMENTS}

The authors express their gratitude to the referees for careful reading and helpful comments on the previous version of this work. The second author was supported by the Scientific Research Project Administration of Akdeniz University.

\section{REFERENCES}

[1] A. Altın and B. Çekim, "Some properties associated with Hermite matrix polynomials." Util. Math., vol. 88, pp. 171-181, 2012.

[2] A. Altın and B. Çekim, "Generating matrix functions for Chebyshev matrix polynomials of the second kind." Hacet. J. Math. Stat., vol. 41, no. 1, pp. 25-32, 2012.

[3] B. Çekim, "A new relation including ${ }_{2} F_{2}$ between Laguerre and Hermite matrix polynomials." $J$. Egyptian Math. Soc., vol. 23, no. 2, pp. 247-250, 2015, doi: 10.1016/j.joems.2014.05.005.

[4] B. Çekim and A. Altın, "A new generalization of Hermite matrix polynomials." Thai J. Math., vol. 13, no. 1, pp. 201-212, 2015.

[5] B. Çekim, A. Altın, and R. Aktas, "Some relations satisfied by orthogonal matrix polynomials." Hacet. J. Math. Stat., vol. 40, no. 2, pp. 241-253, 2011. 
[6] J. H. Conway and R. K. Guy, The Book of Numbers. New York: Springer-Verlag, 1966.

[7] E. Defez and L. Jódar, "Some applications of the Hermite matrix polynomials series expansions." J. Comput. Appl. Math., vol. 99, pp. 105-117, 1998, doi: 10.1016/S0377-0427(98)00149-6.

[8] E. Defez and L. Jódar, "Chebyshev matrix polynomials and second order matrix differential equations." Util. Math., vol. 61, pp. 107-123, 2002.

[9] E. Defez and L. Jódar, "Jacobi matrix differential equation, polynomial solutions, and their properties." Comput. Math. Appl., vol. 48, pp. 789-803, 2004, doi: 10.1016/j.camwa.2004.01.011.

[10] N. Dunford and J. Schwartz, Linear Operators. New York: Interscience, 1963.

[11] H. W. Gould and H. A. T., "Operational formulas connected with two generalizations of Hermite polynomials.” Duke Math. J., vol. 29, pp. 51-63, 1962, doi: 10.1215/S0012-7094-62-02907-1.

[12] N. A. Jedda and A. Ghanmi, "On a class of two-index real Hermite matrix polynomials." Palest. J. Math., vol. 3, no. 2, p. 185, 2014.

[13] L. Jódar and R. Company, "Hermite matrix polynomials and second order matrix differential equations and its applications." J. Approx. Theory, vol. 12, no. 2, pp. 20-30, 1996, doi: 10.1007/BF02836202.

[14] L. Jódar and E. Defez, "Some new matrix formulas related to Hermite matrix polynomials theory." Proceedings of the International Workshop on Orthogonal Polynomials in Mathematical Physics, Leganes, 1996.

[15] L. Jódar and E. Defez, "On Hermite matrix polynomials and Hermite matrix function.” J. Approx. Theory, vol. 4, no. 1, pp. 36-48, 1998, doi: 10.1007/BF02836885.

[16] R. Jódar, L. Company and E. Navarro, "Laguerre matrix polynomials and systems of second order differential equations." Appl. Numer. Math., vol. 15, pp. 53-63, 1994, doi: 10.1016/01689274(94)00012-3.

[17] L. Kargin and V. Kurt, "Some relations on Hermite matrix polynomials." Mathematical and Computational Applications, vol. 18, no. 3, pp. 323-329, 2013, doi: 10.3390/mca18030323.

[18] L. Kargin and V. Kurt, "Modified Laguerre matrix polynomials." Filomat, vol. 10, no. 28, pp. 2069-2076, 2014, doi: 10.2298/FIL1410069K.

[19] L. Kargin and V. Kurt, "On generalized Humbert matrix polynomials." Miskolc Math. Notes, vol. 15, no. 2, pp. 509-524, 2014.

[20] L. Kargin and V. Kurt, "Chebyshev-type matrix polynomials and integral transforms." Hacet. J. Math. Stat., vol. 44, no. 2, pp. 341-350, 2015, doi: 10.15672/HJMS.2015449102.

[21] A. Kishka, Z. M. G. Shehata and M. Abul-Dahab, "The generalized Bessel matrix polynomials." Journal of Mathematical and Computational Science, vol. 2, no. 2, pp. 305-316, 2012.

[22] M. S. Metwally, "Operational rules and arbitrary order two-index two-variable Hermite matrix generating functions.” Acta Math. Acad. Paedagog. Nyhazi., vol. 27, no. 1, pp. 41-49, 2011.

[23] M. T. Metwally, M. S. Mohamed and A. Shehata, "On Hermite-Hermite matrix polynomials." Math. Bohem., vol. 133, no. 4, pp. 421-434, 2008.

[24] M. T. Metwally, M. S. Mohamed and A. Shehata, "Generalizations of two-index two-variable Hermite matrix polynomials.” Demonstr. Math., vol. 42, no. 4, pp. 687-701, 2009.

[25] M. T. Metwally, M. S. Mohamed and A. Shehata, "On pseudo Hermite matrix polynomials of two variables.” Banach J. Math. Anal., vol. 4, no. 2, pp. 169-178, 2010.

[26] E. D. Rainville, Special Functions. New York: The Macmillan Company, 1960.

[27] K. A. M. Sayyed, M. S. Metwally, and R. S. Batahan, "On generalized Hermite matrix polynomials.” Electron. J. Linear Algebra, vol. 10, pp. 272-279, 2003, doi: 10.13001/1081-3810.1113.

[28] A. Shehata, "A new extension of Hermite-Hermite matrix polynomials and their properties." Thai J. Math., vol. 10, no. 2, pp. 433-444, 2012.

[29] A. Shehata, "Connections between Legendre with Hermite and Laguerre matrix polynomials." Gazi University Journal of Science, vol. 28, no. 2, pp. 221-230, 2015. 
[30] A. Shehata, "Some relations on Gegenbauer matrix polynomials." Review of Computer Engineering Research, vol. 2, no. 1, pp. 1-21, 2015, doi: 10.18488/journal.76/2015.2.1/76.1.1.21.

[31] A. Shehata, "Some relations on Laguerre matrix polynomials." Malays. J. Math. Sci., vol. 9, no. 3, pp. 443-462, 2015.

[32] A. Shehata, "Some relations on Konhauser matrix polynomials." Miskolc Math. Notes, vol. 17, no. 1, pp. 605-633, 2016, doi: 10.18514/MMN.2016.1126.

[33] A. Shehata and B. Çekim, "Some relations on Hermite-Hermite matrix polynomials." U.P.B. Sci. Bull., Series A, vol. 78, no. 1, pp. 181-194, 2016.

Authors' addresses

Levent Kargin

Alanya Alaaddin Keykubat University, Akseki Vocational School, TR-07630 Antalya, Turkey

E-mail address: leventkargin48@gmail.com

\section{Veli Kurt}

Akdeniz University, Faculty of Science Department of Mathematics, TR-07058 Antalya, Turkey

E-mail address: vkurteakdeniz.edu.tr 